\title{
Cortical activations during paced finger-tapping applying visual and auditory pacing stimuli
}

\author{
L. Jäncke ${ }^{\mathrm{a}, *}$, R. Loose ${ }^{\mathrm{b}}, \mathrm{K}$. Lutz ${ }^{\mathrm{a}}, \mathrm{K}$. Specht ${ }^{\mathrm{a}, \mathrm{c}}$, N.J. Shah ${ }^{\mathrm{c}}$ \\ ${ }^{a}$ Department of General Psychology, Otto-von-Guericke University Magdeburg, Lennéstraße 6, D-39112 Magdeburg, Germany \\ ${ }^{\mathrm{b}}$ Institute of Psychology, University of Regensburg, Regensburg, Germany \\ ${ }^{\mathrm{c}}$ Institute of Medicine, Research Centre Jülich, Jülich, Germany
}

Accepted 21 March 2000

\begin{abstract}
In order to study neural systems which are involved in motor timing we used whole-brain functional resonance imaging while subjects performed a paced finger-tapping task (PFT) with their right index finger. During one condition, subjects were imaged while tapping in synchrony with tones separated by a constant interval (auditory synchronisation, AS), followed by tapping without the pacing stimulus (auditory continuation, AC). In another condition, subjects were imaged while tapping in synchrony with a visual stimulus presented at the same frequency as the tones (visual synchronisation, VS) followed by a tapping sequence without visual pacing (visual continuation, VC). The following main results were obtained: (1) tapping in the context of visual pacing was generally more variable than tapping in the context of auditory stimuli; (2) during all conditions, a fronto-parietal network was active including the dorsal lateral premotor cortex (dPMC), M1, S1, inferior parietal lobule (LPi), supplementary motor cortex (SMA), the right cerebellar hemisphere, and the paravermial region; (3) stronger activation in the bilateral ventral premotor cortex (vPMC), the left LPi, the SMA, the right inferior cerebellum, and the left thalamus during both auditory conditions (AS and AC) compared to the visual conditions (VS and VC); (4) stronger activation in the right superior cerebellum, the vermis, and the right LPi during the visual conditions (VS and VC); (5) similar activations for the AS and AC conditions; but (6) marked differences between the VS and VC conditions especially in the dorsal premotor cortex (dPMC) and LPi areas; and (7) finally, there were no activations in the auditory and visual cortices when the pacing stimuli were absent. These findings were taken as evidence for a general difference between the motor control modes operative during the auditory and visual conditions. Paced finger tapping in the context of auditory pacing stimuli relies more on brain structures subserving internal motor control while paced finger-tapping in the context of visual pacing stimuli relies on brain structures relying on the subserving processing or imagination of visual pacing stimuli. (C) 2000 Elsevier Science B.V. All rights reserved.
\end{abstract}

Theme: Motor systems and sensorimotor integration

Topic: Control of posture and movement

Keywords: Movement timing; Movement control; Paced finger-tapping; Functional magnetic resonance imaging; Statistical parametric map; SPM

\section{Introduction}

It is generally accepted that motor control involves at least two discernible mechanisms, one of which is concerned with the control of movements in the spatial domain and the other with movement timing. In order to study movement timing, the paced finger-tapping (PFT) task is often used in which subjects produce a series of

*Corresponding author. Tel.: +49-391-6718474; fax: +49-3916711947.

E-mail address: lutz.jaencke@gse-w.uni-magdeburg.de (L. Jäncke). isochronous finger taps, first in synchrony with an external pacing stimulus (synchronisation, S) and then continuing on their own (continuation, C). Some of these studies seem to support the existence of an internal time-keeping system that is thought to be independent of external feedback mechanisms $[48,50]$. Other studies support the contrasting notion that taps are synchronised with the external pacing stimuli at the central level by superimposing in time two sensory codes: a tactile/kinaesthetic code that represents the tap (the afferent movement code) and an auditory code that represents the click (the afferent code that results from the guiding signal). It is thus argued that external feedback 
may play a substantial role for the timing of isochronous taps [2]. Research in patients has linked control of motor timing to a number of motor structures, including the lateral cerebellum and its primary output nucleus, the dentate nucleus [18,19], the basal ganglia [34,38,39], and the supplementary motor area (SMA) [15].

Two recent brain imaging studies employing the PFT paradigm provided results supporting the view that the lateral cerebellar cortex as well as the basal ganglia are involved in the production of timed motor responses. In the study of Rao et al. [40] subjects were imaged applying whole-head functional magnetic resonance imaging (fMRI) while tapping with their right index finger in synchrony with tones separated by two intervals. The results of their study suggest that the internal generation of precisely timed movements is dependent on three interrelated neural systems, one that is involved in explicit timing (putamen, ventrolateral thalamus, SMA), one that mediates auditory sensory memory (inferior frontal gyrus, IFG; superior temporal gyrus, STG), and another that is involved in sensorimotor processing (dorsal dentate nucleus, sensorimotor cortex). A most interesting result of their study is that the right auditory cortex together with the right inferior frontal gyrus is active during the continuation condition in which no explicit auditory pacing stimulus is present. Thus, these results support the notion that in a situation where external sensory input is lacking, a kind of internal generation of the auditory pacing stimuli might take place. A further main result of their study was that SMA as well as cerebellar activation was stronger for the continuation condition, although that was not explicitly tested. In a second brain imaging study (PET technique) related to that topic, Penhune et al. [36] applied auditory and visual pacing stimuli in the context of a PFT paradigm, without, however, using a continuation condition. Their results provide support for a supramodal contribution (independent of stimulus modality) of the lateral cerebellar cortex and cerebellar vermis to the production of a timed motor response. They also gave partial support to the involvement of the basal ganglia in motor timing. In addition, they demonstrated that sensory association areas and the ventromedial frontal cortex were involved in modality-specific encoding of the temporal pacing stimuli. Unfortunately, they did not use a continuation condition which would have allowed to confirm the findings of the Rao et al. study [40]. Furthermore, it would be interesting to know whether the visual cortex is active during a continuation condition, similar to the auditory continuation condition in the study of Rao et al.

Because there are partly conflicting results and some questions unanswered we designed the present study. The research questions are, in particular: (1) to delineate those brain structures which are involved in the control of the PFT task; (2) whether there are differences between auditory and visual pacing conditions in terms of activated brain areas; and (3) to test whether the visual continuation condition is indeed associated with increased activation in visual sensory areas. We thus designed an fMRI experiment in which subjects reproduced an isochronous tapping sequence paced by auditory and visual stimuli and were instructed to continue tapping when these stimuli were discontinued. Similar to the study of Penhune et al. [36] we predicted that if timing is a central process, the activation pattern should be closely similar for the two modalities. In line with the findings of Rao et al. [40], we further predicted that the auditory and visual cortex is active during the auditory and visual continuation conditions.

\section{Materials and methods}

\subsection{Subjects}

Eight healthy, right-handed male adult subjects (age range, 20-32 years) volunteered for this study. All were consistent right-handers as measured by standard handedness tests [37]. Subjects were paid and gave informed consent according to institutional guidelines (Ethics Committee of the University of Düsseldorf).

\subsection{Experimental protocol}

Subjects lay supine on a MRI scanner bed with their right index finger placed on a response keypad, located on the right thigh. The wrist as well as all of the inactive fingers were taped so that they could not participate in the button-press movement. Subjects performed a series of four consecutive activation sessions consisting of four experimental conditions (auditory synchronisation, AS; visual synchronisation, VS; auditory continuation, $\mathrm{AC}$; visual continuation, VC) and a rest condition during which the ambient scanner noise was attended to. In the AS condition, subjects were required to press a key with their right index finger in synchrony with a series of tones separated by a constant interval of $400 \mathrm{~ms}(2.5 \mathrm{~Hz})$. In the VS condition, subjects pressed the key with their right index finger in time with a flicker light presented at the same frequency as the auditory trigger stimuli $(2.5 \mathrm{~Hz})$. The auditory stimulus in the AS condition was a $2000-\mathrm{Hz}$ pure tone presented binaurally at precise intervals using a computer playback system. Sounds were amplified near the scanner using a magnetically shielded transducer system and were delivered to the subject via air conduction through 200-cm paired plastic tubes. The tubes were threaded through tightly occlusive ear inserts that attenuated background scanner noise to $\sim 75 \mathrm{~dB}$ sound pressure level (SPL). Intensity of the tone stimuli averaged $100 \mathrm{~dB}$ SPL [20]. The visual stimulation was delivered via goggles and comprised a 'Ganzfeld-stimulation' of both eyes, making eye movements unlikely. The AC condition immediately followed the AS condition. Subjects were 
instructed to maintain the same tapping rate as in the AS condition (400-ms pacing interval) but without the benefit of the pacing tone. The VC condition immediately followed the VS condition. Subjects were instructed to maintain the same tapping rate as in the VS condition (400-ms pacing interval) but without the benefit of the pacing light.

\subsection{Magnetic resonance imaging}

Subjects were instructed to keep their eyes open throughout the scanning series in order to watch for the visual signals. During the scanning sessions room lights were dimmed. In each session a series of 90 images was acquired. A session comprised 30 of scans for the initial baseline (B, rest), 30 scans for VS or AS, 30 scans for AC or VC. We introduced two visual and auditory conditions and arranged the epochs accordingly (visual condition: B, VS, VC, VS, VC, VS, VC; auditory condition: B, AS, AC, AS, AC, AS, AC) thus requiring 360 scans in total. The stimulation sequence is summarised in Fig. 1. The order of the four conditions was randomised for each subject in order to avoid carry-over effects. The total duration for measuring all functional scans was about $30 \mathrm{~min}$. Functional magnetic resonance (fMRI) imaging was performed on a 1.5-T MRI system (Siemens Magnetom Vision, Erlangen, Germany), equipped with echo planar imaging (EPI) capabilities using the standard head coil for radiofrequency (RF) transmission and signal reception. Sequences with the following parameters were employed: echo planar imaging (EPI), repetition time $(T R)=4.25 \mathrm{~s}$, echo time $(\mathrm{TE})=66 \mathrm{~ms}$, field-of-view $=200 \times 200 \mathrm{~mm}^{2}$, $\alpha=90^{\circ}$, matrix size $=64 \times 64$, voxel size $=3.125 \times 3.125 \times$ $3.3 \mathrm{~mm}^{3}$. Using a mid-sagittal scout image, 32 axial slice positions (0.3-mm inter-slice gap) were oriented parallel to the bi-commissural plane with the uppermost slice aligned $2 \mathrm{~mm}$ above the vertex, thus approximately covering the whole brain. In addition, anatomical images of the entire brain were obtained by using a strongly $\mathrm{T}_{1}$-weighted, gradient-echo pulse sequence in 3D (magnetisation-prepared, rapid acquisition gradient echo, MP-RAGE) with the following parameters: $\mathrm{TR}=11.4 \mathrm{~ms}, \mathrm{TE}=4.4 \mathrm{~ms}, 15^{\circ}$
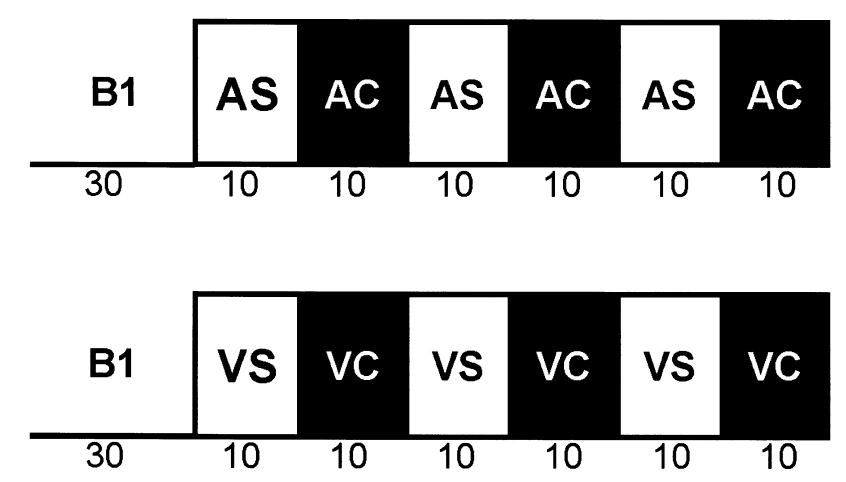

Fig. 1. Illustration of the experimental paradigm. flip angle, field of view $=256 \times 256 \mathrm{~mm}^{2}$, matrix size $=$ $200 \times 256,128$ sagittal slices with $1.33-\mathrm{mm}$ thickness.

\subsection{Image analysis}

Image processing and statistical analysis were carried out using SPM99b software (http://www.fil.ion.ucl.ac.uk/ $\mathrm{spm})$. All volumes were realigned to the first volume, corrected for motion artefacts, mean-adjusted by proportional scaling, co-registered with the subject's corresponding anatomical ( $\mathrm{T}_{1}$-weighted) image, resliced and normalised $\left(4 \mathrm{~mm}^{3}\right)$ into standard stereotaxic space (template provided by the Montreal Neurological Institute [5]), and smoothed using an 8-mm full-width-at-half-maximum Gaussian kernel. Applying a box-car model, adjusted mean images (convolved with the modelled hemodynamic response and eliminating low-frequency noise) were computed for each condition and each subject. These mean images were subjected to a group analysis in the context of a 'random effects procedure' as given by SPM99b. To test the hypothesis about regional condition-specific effects, we applied linear contrasts in the context of the 'General Linear Model' [9-12]. Therefore, we designed predictor variables allowing us to test for main effects (modality: auditory versus visual; pacing condition: synchronisation versus continuation), interactions, and simple effects (i.e., $\mathrm{VS}$ versus VC, AS versus AC). Voxels were identified as significant if they passed the height threshold of $T=3.2$ $(P=0.001$, uncorrected $)$ and a spatial extent threshold of $k=15$ ( $P=0.05$, corrected for multiple comparisons). In order to detect significantly activated voxels in anatomical regions for which we expected activations but which did not reveal any applying the conservative threshold described above, we lowered the height threshold to $T=2.2$ $(P=0.01$, uncorrected $)$. The anatomical regions for which we expected activations are: sensorimotor cortex including M1 and S1, dorsal lateral premotor cortex (dPMD), ventral lateral premotor cortex (vPMC), medial motor wall areas (MMWA: SMA and cingulate motor areas, CMA), inferior parietal lobule (LPi), superior parietal lobule (LPs), auditory cortex on the superior temporal gyrus (GTs), visual cortex in the inferior and medial occipital gyri (GOm, GOi), inferior frontal gyrus (GFi), cerebellar hemisphere, cerebellar vermis and paravermial region (CB), basal ganglia (BG), and thalamus (TH) $[6,16,36,40]$.

In order to strengthen our statistical analysis for the between-condition comparisons, we only applied the designed contrasts to voxels which survived a 'masking' contrast. For instance, for the contrast VS $>$ VC we used as masking contrast VS >rest with a threshold of $P=0.05$. The resulting set of voxel values for each contrast constitutes a statistical parametric map of the $T$ statistic $(\operatorname{SPM}\{T\})$. The activated voxels surviving this procedure were superimposed on the averaged stereotaxically normalised high-resolution MR-anatomical scans as well as on a standard MNI brain in order to identify the anatomical 
regions. In order to allow a precise mapping of the activation peaks, we tabulated peak activations and subpeaks arising from the different contrast.

\section{Results}

\subsection{Performance data}

Table 1 displays the mean intertap intervals (ITI) for all conditions. ITIs that exceeded $50 \%$ of the target interval duration were excluded from the reaction time data. This occurred in less than $1 \%$ of all trials and mostly at the beginning of a condition, i.e., reflecting a problem of starting the PFT task in time. As can be seen from Table 1, ITIs precisely matched the prescribed pacing rhythm. A two-way analysis of variance with repeated measurements on both factors (modality: auditory versus visual; pacing: synchronisation versus continuation) revealed only a significant main effect for the pacing factor $(F=6.7, \mathrm{df}=1.7$, $P=0.036$, ETA $2=0.48$ ) depending on slightly larger ITIs for the synchronisation tasks (mean difference between $\mathrm{S}$ and $\mathrm{C}$ conditions, $11.6 \mathrm{~ms}$ ). However, within-subject ITI variability was larger for the visual conditions (S.D.: for V, 36.8; and for A, 6.5) indicating differences with respect to the control of the visual and auditory tasks. ITI variability as measured by the coefficient of variation (S.D. $\times 100 /$ mean ITI) was also subjected to a two-way analysis of variance with repeated measurements on both factors. This analysis revealed a trend towards a significant interaction between both factors $\left(F=2.9, \mathrm{df}=1,7, P=0.18, \mathrm{ETA}^{2}=\right.$ $0.24)$. This interaction was due to a larger increase of ITI variability from the $\mathrm{S}$ to the $\mathrm{C}$ condition for the visual modality. Variability results are roughly similar to those found by Rao et al. [40]. For the S conditions, we also computed differences between the occurrence of the pacing stimulus and the tap to test whether we could confirm a 'negative lag' effect found in previous PFT studies

Table 1

Mean intertap interval (ITI in ms) and ITI variability (coefficient of variation) for the four tapping conditions (VS, visual synchronisation; VC, visual continuation; AS, auditory synchronisation; AC, auditory continuation) ${ }^{\mathrm{a}}$

\begin{tabular}{llr}
\hline & ITI $(\mathrm{ms})$ & Variability \\
\hline VS & $403.6 \pm 34.4$ & $10.9 \pm 5.8$ \\
VC & $400.3 \pm 39.7$ & $12.1 \pm 3.1$ \\
AS & $392.5 \pm 10.7$ & $10.9 \pm 6.9$ \\
AC & $378.8 \pm 10.1$ & $9.6 \pm 4.4$ \\
S (VS+AS) & $398.0 \pm 14.6$ & $10.9 \pm 5.6$ \\
C (VC+AC) & $389.6 \pm 21.4$ & $10.8 \pm 2.7$ \\
V (VS+VC) & $401.9 \pm 38.8$ & $11.5 \pm 4.1$ \\
A (AS+AC) & $385.7 \pm 6.5$ & $10.3 \pm 4.9$ \\
\hline
\end{tabular}

${ }^{a}$ Mean ITIs and ITI variability coefficients are also given for the main effects of sensory conditions (auditory [A] versus visual [V]) and pacing condition (synchronisation $[\mathrm{S}]$ and continuation [C]). Corresponding standard deviations are also presented.
[2,23,32]. We did indeed find significant 'negative lags', i.e., with taps occurring before the pacing stimuli (mean (S.D.: auditory, $48.2 \mathrm{~ms}, t(7)=2.6, P=0.03$; visual, 24.3 ms, $t(7)=5.3 ; P<0.001$, Table 1). There was a trend towards a slightly larger 'lag-effect' for the AS condition ( $t$-test for dependent samples: $t=2.0, \mathrm{df}=7, P=0.08$, twosided test).

\subsection{Neurophysiology}

As can be seen in Table 2, there were significant hemodynamic responses in the left M1, S1, vPMC, SMA, ventro-lateral thalamus, the right inferior cerebellum, the vermis, and bilaterally in the LPi during the auditory conditions. Thus, we revealed activations relative to rest in all regions as expected with the exception of inferior frontal gyrus activation. The AS condition, however, revealed hemodynamic responses in the left auditory cortex located on the superior temporal gyrus (anterior and posterior GTs), while there was no activation in this area during the AC condition. There is currently no consensus according to the exact location of vPMC. In the macaque brain, there is a more ventral premotor area identified as ventral premotor area. This lies in and below the spur of the arcuate sulcus in area F4/F5 [31]. It is located at the level of the frontal eye-fields as defined by microstimulation [3]. In PET studies, the frontal eye-fields have been identified as lying from 40 to $56 \mathrm{~mm}$ above the AC-PC line $[1,8]$. In human brain imaging studies the vPMC has been identified as being located on the lateral surface at the level of the lower slices through the frontal eye-fields. This roughly corresponds with slice position ranging from 36 to $46 \mathrm{~mm}$ above the AC-PC line. Activations in these slice positions (38 and $44 \mathrm{~mm}$ ) were found by Kawashima et al. [22] while subjects learned to reach for targets. In addition, Grafton et al. [13] scanned subjects while they performed a visual tracking task, and there was activation at a height of $36 \mathrm{~mm}$ above the AC-PC line. Some authors have identified a ventral premotor area more ventrally than the above described area lying in the opercular cortex [49]. Finally, it is possible that a part of vPMC extends into area 44 , the region anterior to opercular area 6 . Activations in this area have been reported when subjects imagine movements [49], prepare a movement [24], or imitate a movement $[7,45]$. In our study, we assigned activation in premotor areas located $34-48 \mathrm{~mm}$ above the AC-PC line as vPMC and activations above $48 \mathrm{~mm}$ as dPMC. Thus, we argue that the auditory conditions revealed bilateral activations in vPMC (Table 2: $x=-52, y=-8, z=44$; Table 4: $x=-48, y=-4, z=44 ; x=64, y=0, z=32)$.

The analysis of the visual conditions, VS and VC, revealed partly similar results as for the auditory conditions in terms of demonstrating activations in the hypothesised regions (Table 3 ). However, we also found activations in the left and right LPi, as well as in LPs. Interestingly, the cerebellar activation for both visual 
Table 2

Brain areas activated during the auditory synchronisation (AS) and continuation (AC) conditions ${ }^{\mathrm{a}}$

\begin{tabular}{|c|c|c|c|c|c|c|c|c|}
\hline & Region & $x$ & $y$ & $z$ & AS & $\mathrm{AC}$ & $\mathrm{AS}>\mathrm{AC}$ & $\overline{\mathrm{AC}>\mathrm{AS}}$ \\
\hline Premotor and & L vPMC & -52 & -8 & 44 & 6.8 & 6.8 & - & - \\
\hline \multirow[t]{4}{*}{ somatomotor cortex } & L M1 & -44 & -20 & 52 & 6.5 & 6.7 & - & - \\
\hline & L M1 & -56 & -24 & 44 & 5.7 & 4.8 & - & - \\
\hline & L S1 & -32 & -28 & 64 & 5.2 & 5.3 & - & - \\
\hline & L SMA & -4 & -4 & 52 & 5.9 & 6.7 & - & - \\
\hline \multirow[t]{4}{*}{ Temporal lobe } & L GTs & -64 & -28 & 16 & 4.3 & $2.2^{\mathrm{b}}$ & - & - \\
\hline & L GTs & -64 & -36 & 28 & 3.5 & 3.9 & - & - \\
\hline & L GTs & -60 & -16 & 8 & $2.5^{\mathrm{b}}$ & $-2.8^{\mathrm{b}}$ & 5.2 & - \\
\hline & R GTs & 60 & -20 & 4 & $2.2^{\mathrm{b}}$ & -3.7 & 5.9 & - \\
\hline \multirow[t]{2}{*}{ Parietal lobe } & L LPi & -48 & -36 & 52 & 3.5 & 3.6 & - & - \\
\hline & R LPi & 56 & -32 & 32 & 3.6 & 3.6 & - & - \\
\hline \multirow[t]{3}{*}{ Cerebellum } & $\mathrm{R} C B$ inferior & 12 & -64 & -48 & 5.1 & 6.4 & - & - \\
\hline & $\mathrm{R} C B$ vermis & 4 & -56 & -12 & 3.7 & 4.5 & - & - \\
\hline & R CB superior & 16 & -48 & -24 & - & 3.2 & - & - \\
\hline Thalamus & $\mathrm{L}$ vl & -16 & -20 & -4 & 3.4 & $2.2^{\mathrm{b}}$ & - & - \\
\hline
\end{tabular}

${ }^{a}$ The table gives the $t$ values of the maxima from the main effects (AS, AS $>$ rest; AC, AC $>$ rest) as well as the maxima for differences between the different conditions (AS $>\mathrm{AC}$ and $\mathrm{AC}>\mathrm{AS}$ ). All $t$ values are associated with 75 degrees of freedom. $t \geq 4.9, P \leqslant 0.05$ (corrected); $t \geq 3.2$ and $t<4.9$, $P \leqslant 0.001$ (uncorrected); $t \leqslant 2.2, P \leqslant 0.01$ (uncorrected). $x, y, z:$ MNI coordinates, dPMC, dorsal premotor cortex; vPMC, ventral premotor cortex; M1, primary motor cortex; S1, primary somatosensory cortex; SMA, supplementary motor area; GTs, superior temporal gyrus; LPi, inferior parietal lobe; CB, cerebellum; vl, ventrolateral; $\mathrm{R}$, right; $\mathrm{L}$, left.

${ }^{\mathrm{b}}$ Voxels where a lowered significance threshold of $t=2.2$ was adopted in order to detect small activations in anatomical regions which were hypothesised to reveal significant activations for this task

conditions were located in the right superior cerebellum $(x=16, y=-48, z=-24)$ while the cerebellar activations for the auditory conditions were located in the inferior cerebellum. Interestingly, there were right-sided vPMC $(x=58, y=8, z=40)$ and bilateral activations in dPMC $(x=-44, y=-4, z=56$ and $x=44, y=-4, z=56)$ during the visual conditions.

Comparing the auditory (A) and visual conditions (V) (main effect analysis for modality) revealed stronger signal increases bilaterally in the vPMC (Fig. 2A,B), SMA (Fig. 2C), the right inferior cerebellum (Fig. 3A), the left thalamus (Fig. 3B), and the left LPi (Fig. 3C) (see also Table 4). It is interesting to note that the activation within the SMA was located at an inferior position $(z=48)$ slightly above the cingulate sulcus (see Fig. 2C). Interestingly, the auditory conditions evoked stronger activation in

Table 3

Brain areas activated during the visual synchronisation (VS) and continuation (VC) conditions $^{\mathrm{a}}$

\begin{tabular}{|c|c|c|c|c|c|c|c|c|}
\hline & Region & $x$ & $y$ & $z$ & VS & $\mathrm{VC}$ & $\mathrm{VS}>\mathrm{VC}$ & $\mathrm{VC}>\mathrm{VS}$ \\
\hline Premotor and & $\mathrm{L} \mathrm{dPMC}$ & -44 & -4 & 56 & 5.4 & 6.8 & - & - \\
\hline \multirow[t]{6}{*}{ somatomotor cortex } & L M1 & -44 & -16 & 56 & 5.4 & 6.9 & - & - \\
\hline & M SMA & 0 & 0 & 60 & 4.8 & 4.5 & - & - \\
\hline & $\mathrm{R}$ dPMC & 44 & -4 & 56 & 4.1 & - & 3.7 & - \\
\hline & $\mathrm{R}$ vPMC & 56 & 8 & 40 & 5.4 & - & 3.2 & - \\
\hline & $\mathrm{L}$ dPMC & -32 & -12 & 60 & - & 4.6 & - & 4.0 \\
\hline & L M1 & -52 & -20 & 44 & - & 4.9 & - & 3.5 \\
\hline \multirow[t]{6}{*}{ Parietal Lobe } & L S1 & -44 & -32 & 52 & 3.2 & 6.2 & - & $2.9^{\mathrm{b}}$ \\
\hline & L LPi & -56 & -32 & 44 & 5.2 & 8.0 & - & $2.8^{\mathrm{b}}$ \\
\hline & R LPi & 40 & -56 & 48 & 5.0 & 4.3 & - & - \\
\hline & R LPi & 52 & -40 & 48 & 5.7 & 6.0 & - & - \\
\hline & R LPi & 48 & -48 & 52 & 4.1 & 5.2 & - & - \\
\hline & R LPs & 20 & -52 & 68 & - & 3.2 & - & 3.4 \\
\hline Cerebellum & $\mathrm{R} \mathrm{CB}$ & 16 & -48 & -24 & 5.3 & 6.0 & - & - \\
\hline \multirow[t]{2}{*}{ Occipital ortex } & L GOi & -12 & -88 & 0 & 8.4 & -4.1 & 12.9 & - \\
\hline & R GOi & 4 & -80 & 4 & 8.3 & -4.1 & 12.2 & - \\
\hline
\end{tabular}

${ }^{a}$ The table gives the $t$ values of the maxima from the main effects (VS, VS $>$ rest; VC, VC $>$ rest) as well as the maxima for differences between the different conditions (VS $>\mathrm{VC}$ and $\mathrm{VC}>\mathrm{VS}$ ). All $t$ values are associated with 75 degrees of freedom. $t \geq 4.9: P \leqslant 0.05$ (corrected); $t \geq 3.2$ and $t>4.9$, $P \geq 0.001$ (uncorrected); $t \leqslant 2.2, P \leqslant 0.01$ (uncorrected). $x, y, z:$ MNI coordinates, dPMC, dorsal premotor cortex; vPMC, ventral premotor cortex; M1, primary motor cortex; S1, primary somatosensory cortex; SMA, supplementary motor area; GTs, superior temporal gyrus; LPi, inferior parietal lobe; CB, cerebellum; vl, ventrolateral; R, right; L, left.

${ }^{\mathrm{b}}$ Voxels where a lowered significance threshold of $t=2.2$ was adopted in order to detect small activations in anatomical regions which were hypothesised to reveal significant activations for this task. 
A)
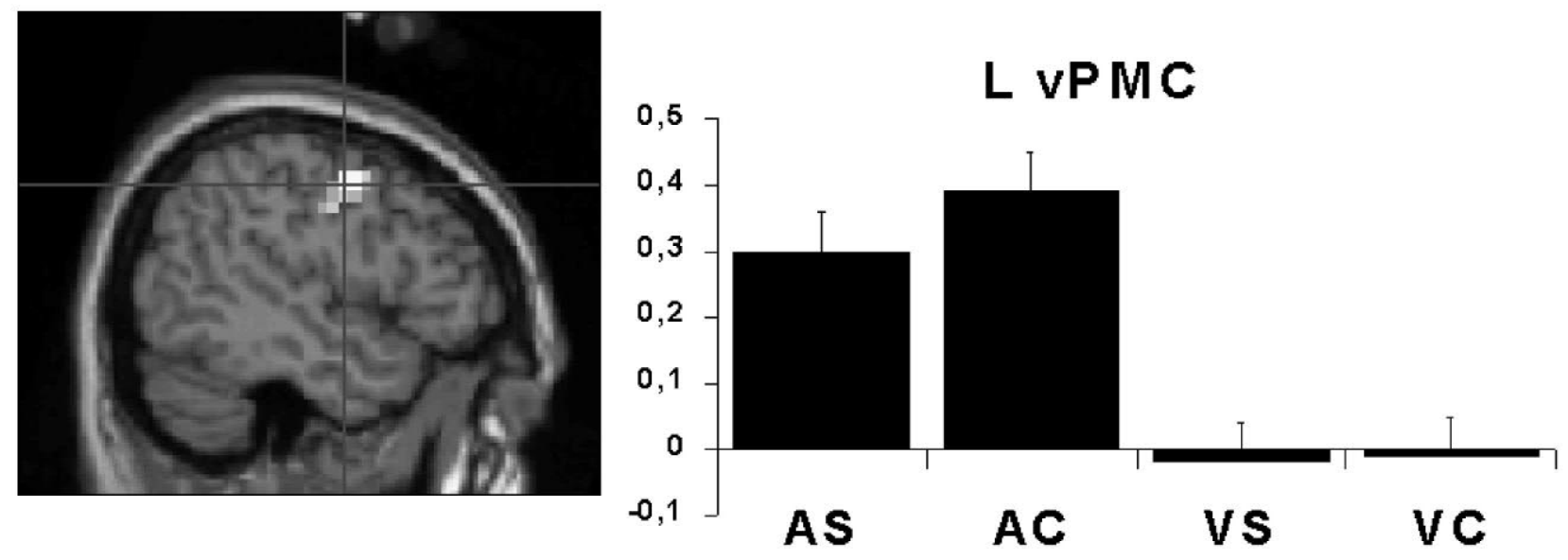

B)
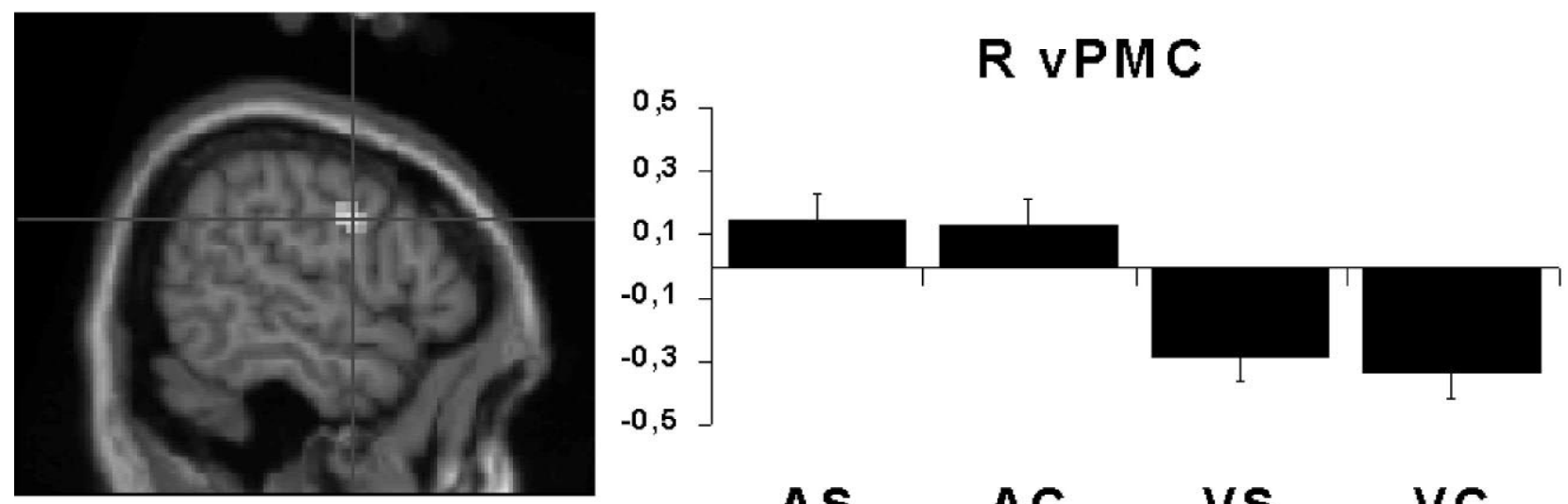

C)
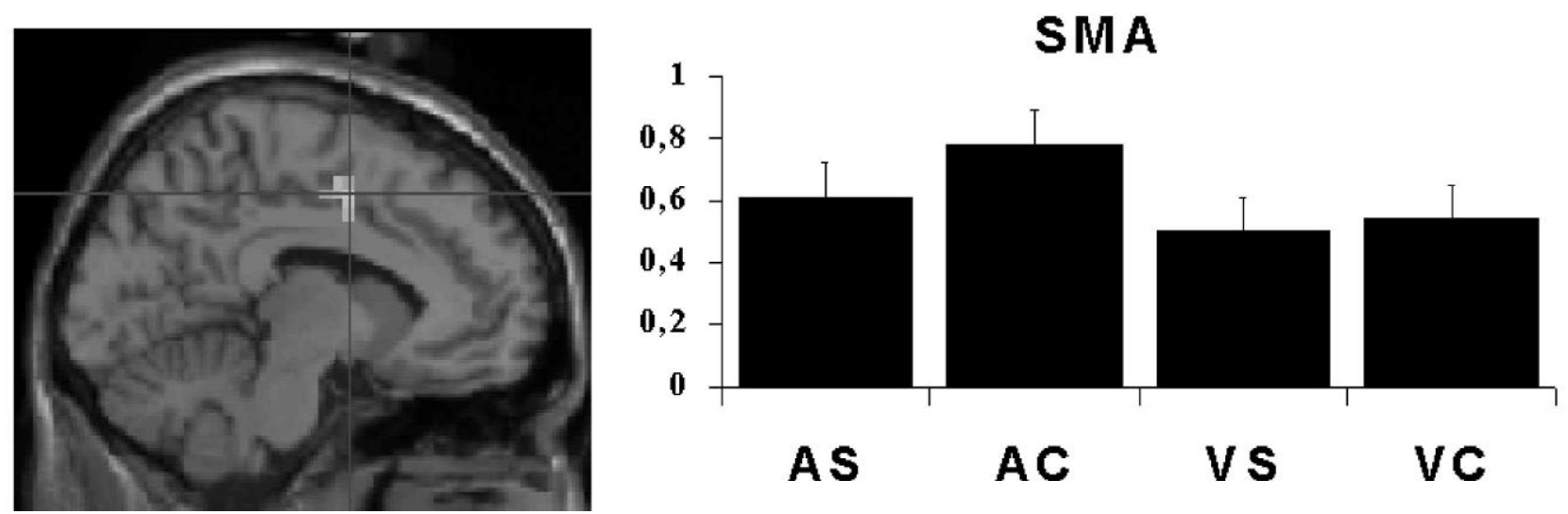

Fig. 2. (A-C) Anatomical rendering (left) of significant increases of hemodynamic responses for the main effect 'modality' (auditory $>$ visual) and the effect sizes (standard errors as vertical bars) for each condition at this particular local maximum (right). (A) Left ventral premotor cortex $(-48,-4,44)$; (B) right ventral premotor cortex $(64,0,32)$; (C) left SMA $(-8,0,48)$. The $\operatorname{SPM}(T)$ for the relevant contrast has been thresholded at $P<0.001$ and superimposed on a representative brain used as template for the spatial normalisation of the images. 
A)
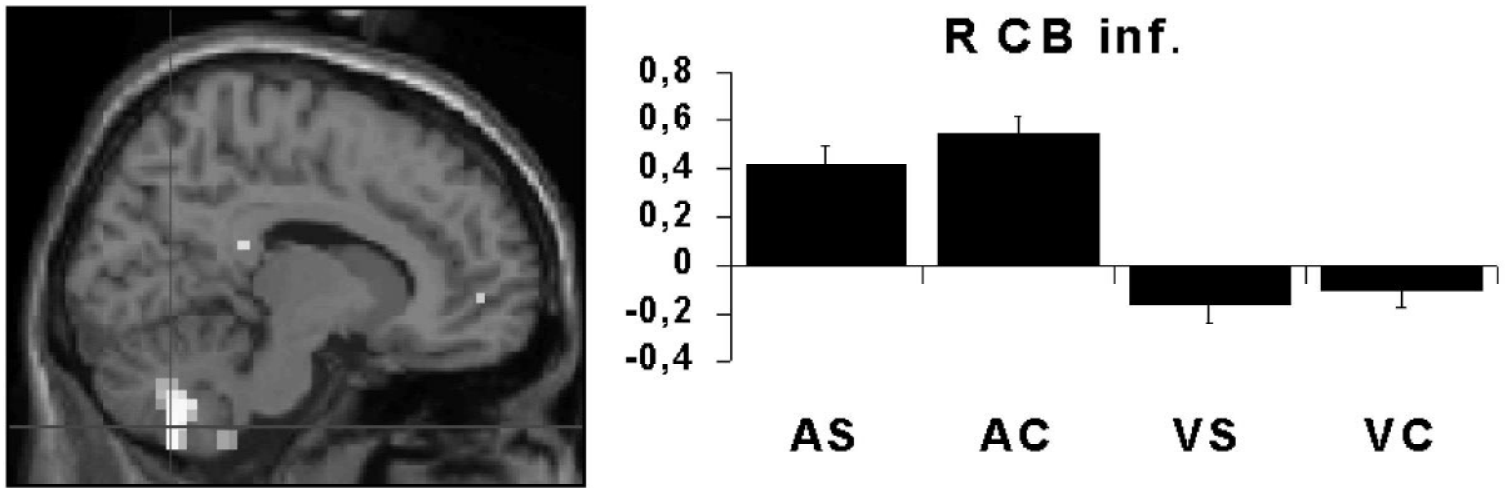

\section{AS AC VS VC}

B)
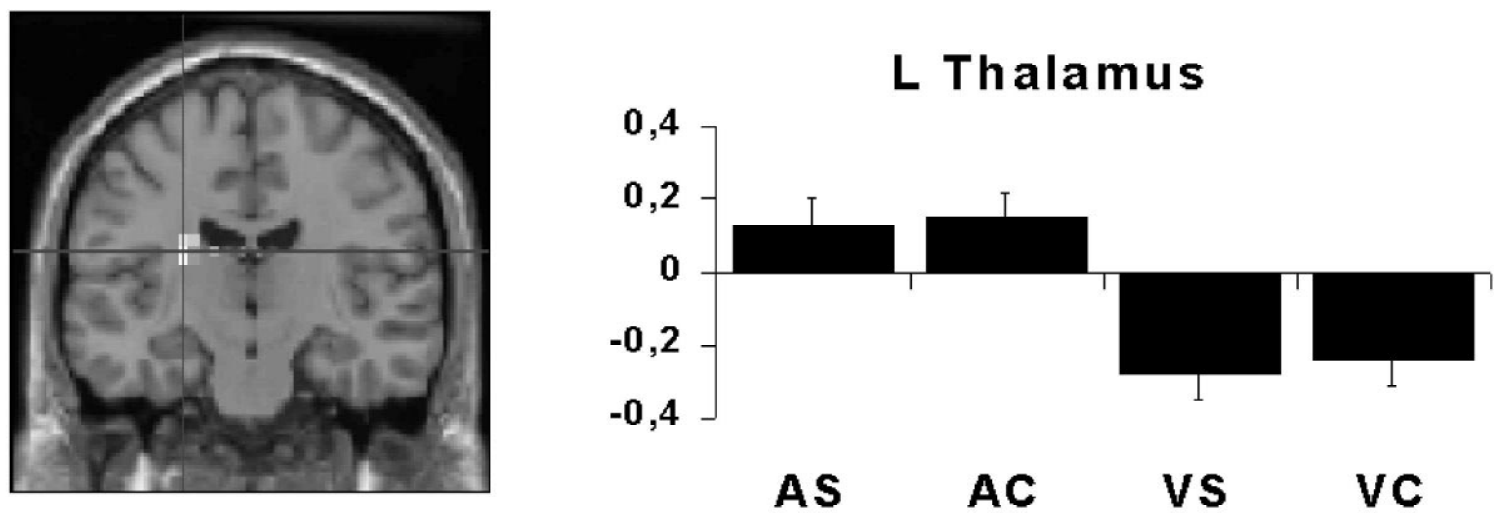

C)
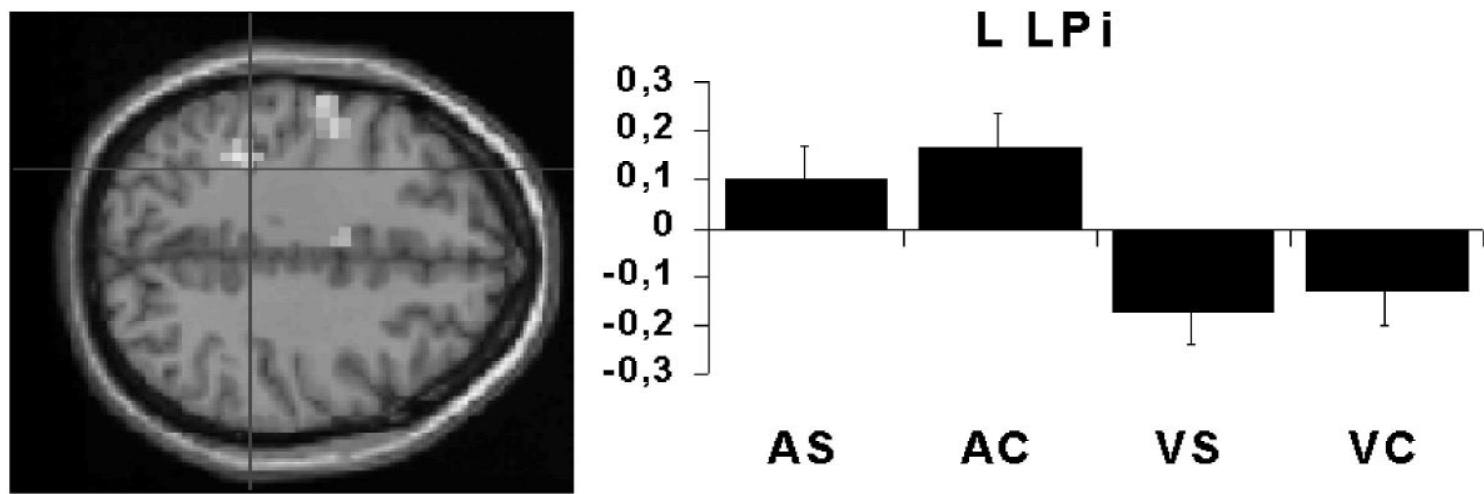

Fig. 3. (A-C) Anatomical rendering (left) of significant increases of hemodynamic responses for the main effect 'modality' (auditory $>$ visual) and the effect sizes (standard errors as vertical bars) for each condition at this particular local maximum (right). (A) Right inferior cerebellum (12, $-64,-48$ ); (B) left thalamus $(-24,-16,20)$; (C) left inferior parietal lobe $(-36,-40,40)$. The $\operatorname{SPM}(T)$ for the relevant contrast has been thresholded at $P<0.001$ and superimposed on a representative brain used as template for the spatial normalisation of the images.

the inferior cerebellum $(x=12, y=-64, z=-48)$ located at the $\mathrm{H}$ VIIB, H IIXA, and $\mathrm{H}$ VIII according to the nomenclature of Larsell and Jansen [30]. The visual conditions evoked on average stronger activations within the vermis (Fig. 4A), the right LPi (Fig. 4B), the right GOi (Fig. 4C), and the right superior cerebellum (Fig. 4D). In a further step we searched for a general difference between the synchronisation and continuation conditions across 
Table 4

Brain areas activated during the auditory (A) and visual (V) conditions ${ }^{\text {a }}$

\begin{tabular}{rlrrrr}
\hline & Anatomical region & \multicolumn{1}{c}{$x$} & \multicolumn{1}{c}{$z$} & $T$ \\
\hline A $>$ V & R CB inferior & 12 & -64 & -48 & 5.9 \\
& L Thalamus & -24 & -16 & 20 & 5.0 \\
& L vPMC & -48 & -4 & 44 & 4.6 \\
& R vPMC & 64 & 0 & 32 & 4.3 \\
& L LPi & -36 & -40 & 40 & 3.8 \\
& SMA & -8 & 0 & 48 & 3.3 \\
V $>$ A & R GOi & 60 & -52 & -4 & 4.6 \\
& Vermis & -4 & -68 & -24 & 4.6 \\
& R CB superior & 40 & -52 & -28 & 3.7 \\
& R LPI & 56 & -36 & 48 & 5.3 \\
\hline
\end{tabular}

${ }^{a}$ The voxels show levels of significance above a threshold of $t(75)=3.2$ $(P<0.001$, uncorrected). L and $\mathrm{R}$, left and right; vPMC, ventral lateral premotor area; $\mathrm{CB}$, cerebellum; $\mathrm{LPi}$, inferior parietal lobule; SMA, supplementary motor area. $\mathrm{V}=(\mathrm{VS}+\mathrm{VC}) / 2>$ rest; $\mathrm{A}=(\mathrm{AS}+\mathrm{AC}) / 2>$ rest.

both stimulus modalities (main effect analysis for pacing conditions). However, we did not find any consistent difference between these conditions.

As can be seen on Table 2, there were no substantial differences between the paced and non-paced auditory conditions (AS and AC) applying the chosen threshold criterion. We only found a substantial difference for the hemodynamic responses bilaterally in the auditory cortex roughly in the vicinity of Heschl's gyrus $(x=-60, y=-$ $16, y=8 ; x=60, y=20, z=4)$. Interestingly, this difference was found at locations where there were substantial deactivations during the AC condition (Fig. 5A,B). Comparing the VS with the $\mathrm{VC}$ condition revealed signal increases during the VS condition in the visual cortices bilaterally (Fig. 5C,D). There were no hemodynamic responses in these areas during the $\mathrm{VC}$ condition. Besides, these differences in the auditory and visual areas during the synchronisation and continuation conditions, there were further marked differences. First, there were stronger hemodynamic responses in the right dPMC (Fig. 6A), the right vPMC (Fig. 6B), and bilaterally in the fusiform gyrus (GF) (Fig. 6C,D) for the VS condition. The VC condition on the other hand turned out to evoke stronger responses in the left dPMC (Fig. 7A), the left MI (Fig. 7B), the left SI (Fig. 7C), and the left LPs (Fig. 7D).

\section{Discussion}

The present experiment was designed to delineate those brain structures which are involved in the control of paced finger-tapping in the context of visual or auditory pacing stimuli both when the pacing stimulus was present (synchronisation) or absent (continuation). The performance measures obtained in the present study were basically similar to those found in previous experiments applying the same task $[2,23,32,40]$. In summary, we found a 'negative lag-effect' during the synchronisation conditions with finger taps slightly preceding the pacing stimuli for about 30-50 ms. In addition, we also found larger intertap interval variability for the continuation conditions compared to the synchronisation conditions. Finally, this variability was generally larger for the visual conditions, suggesting different motor control processes for the auditory and visual as well as for the synchronisation and continuation conditions. With respect to the neurophysiological activations, our results are principally in agreement with the findings of earlier brain imaging studies using the PFT task in demonstrating that during the paced and non-paced conditions a network is active that includes M1, S1, SMA, as well as cerebellar areas [36,40]. A new finding of the present study, compared to those studies which also applied the PFT task, are activations in a fronto-parietal network comprising dPMC, vPMC, and LPi as well as activations within the left thalamus which were differently activated in the visual (VS and VC) and auditory (AS and AC) conditions. In particular, we uncovered the following new findings: (1) stronger activation in the bilateral vPMC, the left LPi, the SMA, the right inferior cerebellum, and the left thalamus during both auditory conditions (AS and AC) compared to the visual conditions (VS and VC); (2) stronger activation in the right superior cerebellum, the vermis, and the right LPi during the visual conditions (VS and VC), (3) similar activations for the AS and AC conditions; but (4) marked differences between the VS and VC conditions especially in dPMC and LPi areas; and (5) finally, there were no activations in the auditory and visual cortices when the pacing stimuli were absent thus suggesting that internal time-keeping is not confined to the imagination of pacing signals by means of activating these sensory areas. In the following, we will discuss the neurophysiological findings and how they relate to published findings of the neurophysiological and psychological literature.

\subsection{Frontoparietal network}

According to earlier hypotheses, dPMC and vPMC are specifically activated by movements that are guided by sensory information as opposed to movements that are carried out with no sensory control [13,22,35]. In nonhuman primates, a ventral and a dorsal frontoparietal network have been implicated for visuomotor transformations of movements, with vPMC and dPMC as the prerolandic components of this system. The ventral system including the ventral intraparietal area (VIP) bordering the LPi and vPMC is suggested to code target localisation in peripersonal space in body-part centered coordinates [14]. The dorsal system, comprising of the dPMC and the medial intraparietal area (MIP) bordering the LPi, is thought to be involved in the control of motor functions subserving visuomotor transformations by reference to an external, visuospatial reference frame. However, activations in vPMC have been reported during complex motor 

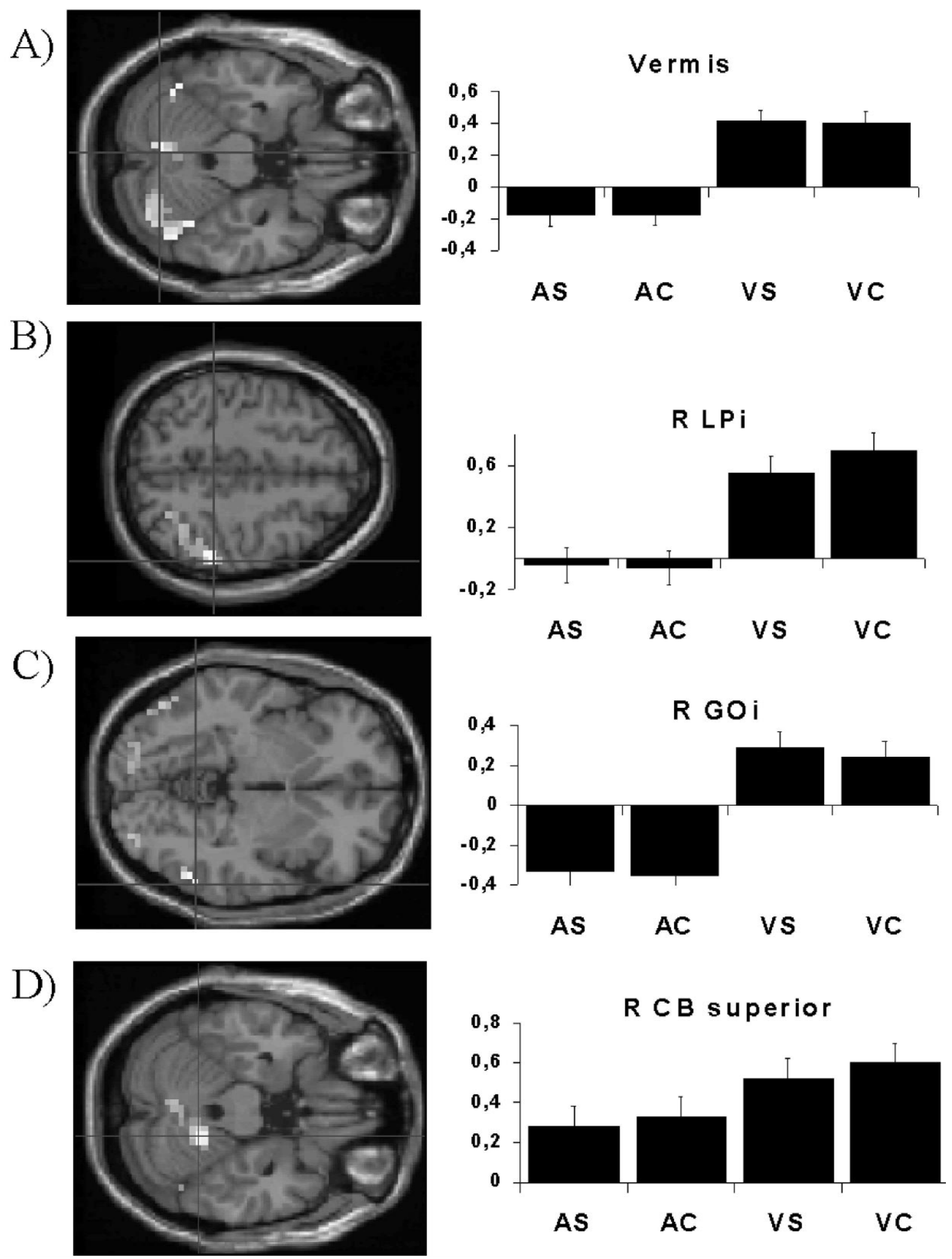

Fig. 4. (A-D) Anatomical rendering (left) of significant increases of hemodynamic responses for the main effect 'modality' (visual $>$ auditory) and the effect sizes and (standard errors as vertical bars) for each condition at this particular local maximum (right). (A) Vermis $(-4,-68,-24)$; (B) right inferior parietal lobule $(56,-36,48)$; (C) right inferior occipital gyrus $(60,-52,-4)$; (D) right superior cerebellum $(40,-52,28)$. The SPM(T) for the relevant contrast has been thresholded at $P<0.001$ and superimposed on a representative brain used as template for the spatial normalisation of the images. 

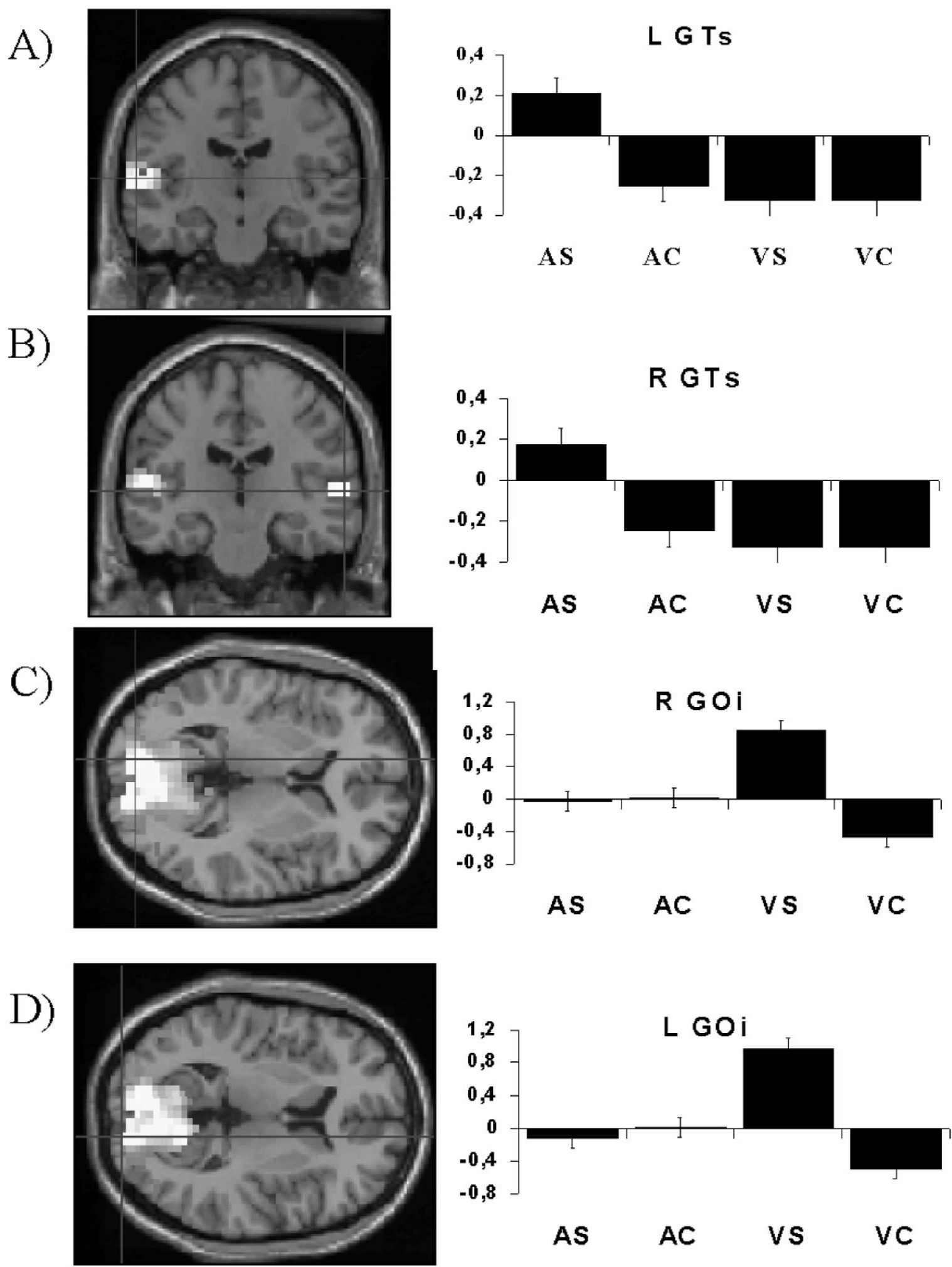

Fig. 5. (A-D) Anatomical rendering (left) of significant increases of hemodynamic responses for the interaction effects ([AS $>(A C+V S+V C)]$ and [VS $>(\mathrm{VC}+\mathrm{AS}+\mathrm{AC})]$ ) and the effect sizes and (standard errors as vertical bars) for each condition at this particular local maximum (right). (A) Left superior temporal gyrus (transversal gyrus) $(-64,-16,8)$; (B) right superior temporal gyrus (transversal gyrus) $(60,-20,4)$; (C) right inferior occipital gyrus $(4,-80,4)$; (D) left inferior occipital gyrus $(-12,-88,0)$. The $\operatorname{SPM}(T)$ for the relevant contrast has been thresholded at $P<0.001$ and superimposed on a representative brain used as template for the spatial normalisation of the images. 

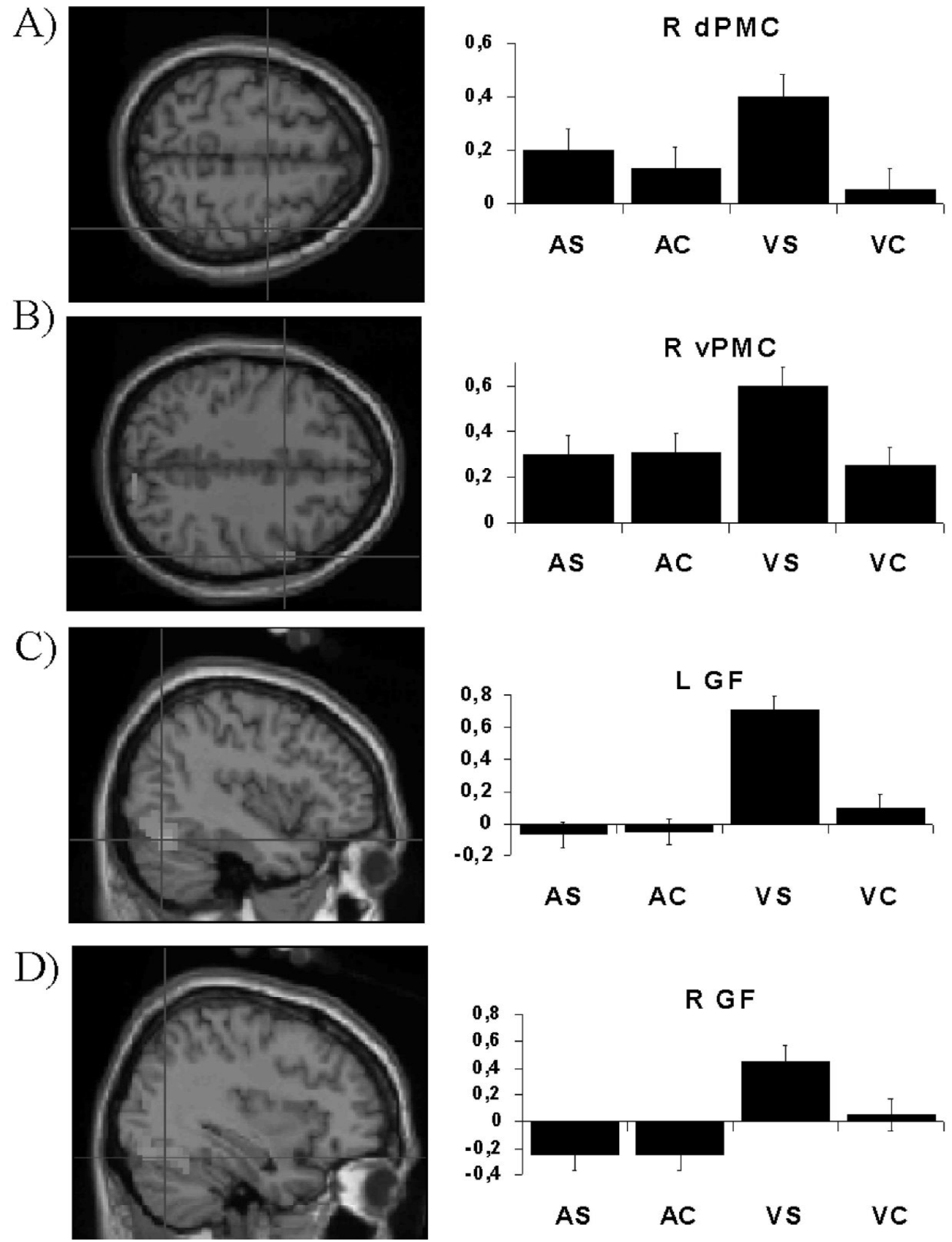

Fig. 6. (A-D) Anatomical rendering (left) of significant increases of hemodynamic responses for the interaction effects $([\mathrm{VS}>(\mathrm{VC}+\mathrm{AS}+\mathrm{AC})])$ and the effect sizes (standard errors as vertical bars) for each condition at this particular local maximum (right). (A) Right dorsal premotor cortex (44, $-4,56)$; (B) right ventral premotor cortex $(56,8,40)$; (C) left fusiform gyrus $(-40,-60,-16)$; (D) right fusiform gyrus $(38,-58,-14)$. The SPM(T) for the relevant contrast has been thresholded at $P<0.001$ and superimposed on a representative brain used as template for the spatial normalisation of the images. 

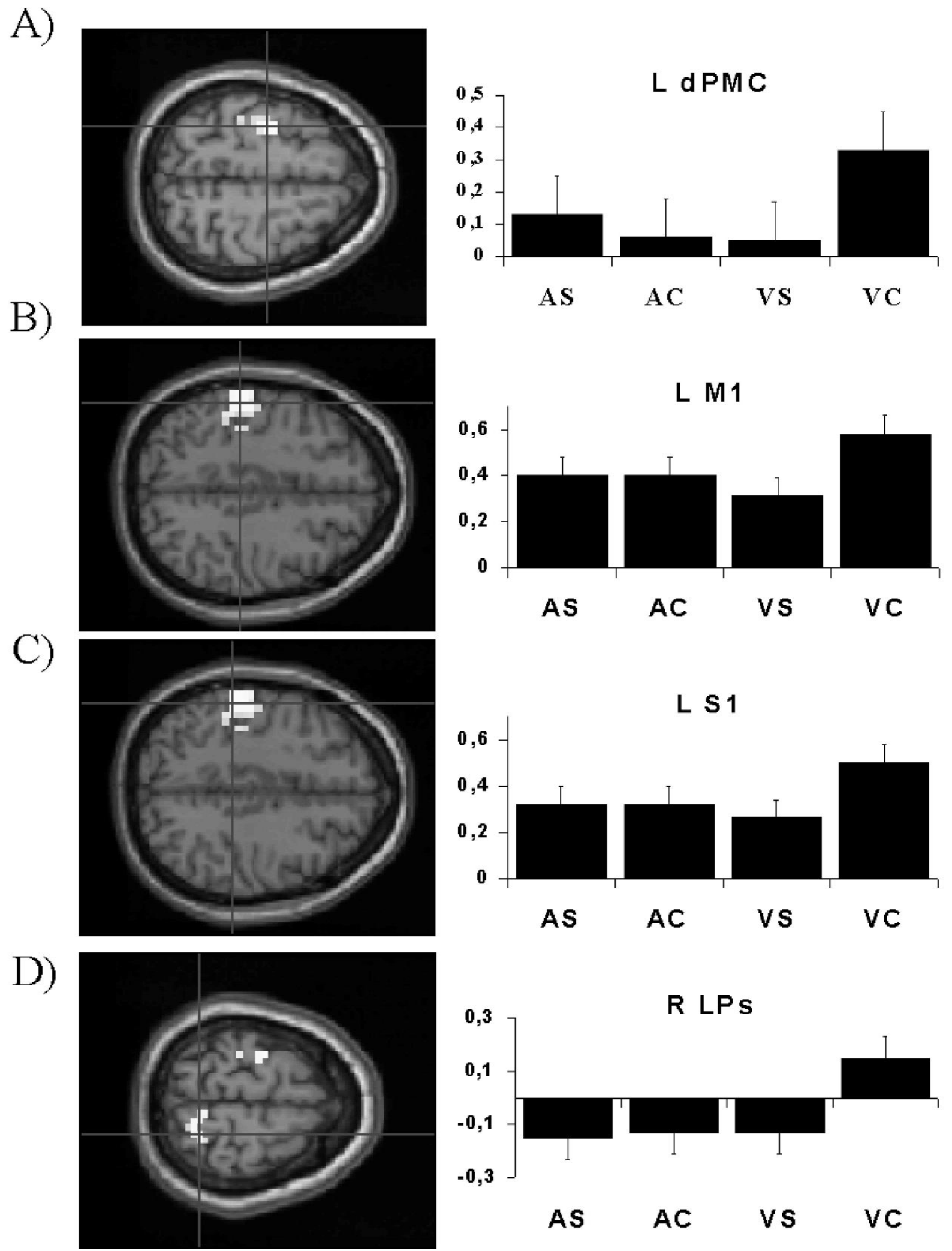

Fig. 7. (A-D) Anatomical rendering (left) of significant increases of hemodynamic responses for the interaction effects (VC $>$ VS) and the effect sizes and (standard errors as vertical bars) for each condition at this particular local maximum (right). (A) Left caudal dorsal premotor cortex ( $-32,-12,60)$ right dorsal premotor cortex $(44,-4,56)$; (B) left M1 $(-52,-20,44)$; (C) left S1 $(-44,-32,52)$; (D) right superior parietal lobule $(20,-52,68)$. The $\operatorname{SPM}(T)$ for the relevant contrast has been thresholded at $P<0.001$ and superimposed on a representative brain used as template for the spatial normalisation of the images. 
tasks like grasping or reaching which are visually guided [25-29,42,43]. Although there is no general consensus with respect to the differential roles of dPMC and vPMC in the visual guidance of movements, there are more and more data suggesting that the vPMC plays an important role in motor learning and in the recalibration of visual and motor coordinates. A more ventral and rostral part of the vPMC is also suggested to be involved in the imagination of complex movements [49], preparation of imitated movements [24], or imitation of movements in general $[41,44,45]$.

Why are the auditory conditions more prone to evoke activations in the vPMC as compared to the visual conditions? From the current state of knowledge it is difficult to give a plausible answer. One possibility would be that the vPMC area is specifically involved when higher-order sensory-motor associations are necessary for the motor task, especially when prediction, anticipation, or imagination is necessary. Anticipation and prediction are important psychological functions necessary to achieve precise finger tapping timing in the context of PFT tasks. As can be seen from the performance data, the tapping performance is much better for the auditory than for the visual conditions, suggesting that these processes might be applied more efficiently to the auditory tapping tasks. On the other hand, it might also be that the ventral pathway is activated because there is ongoing motor learning and recalibration of sensory and motor codes.

A different picture emerged for the visual conditions. During both visual conditions there were left-sided dPMC activations, but only the VS condition additionally evoked activation on the right $\mathrm{APMC}$, while the VC condition evoked stronger activation on a caudal part of the left dPMC together with stronger activations in left LPi and the right LPs. Thus, there is a clear difference between the visual and auditory conditions as well as between the VS and VC conditions in terms of the participating frontoparietal brain structures. We suggest that the dPMC areas are activated when subjects perform visual conditional tasks irrespective of whether they have to perform movements in the spatial or time domain. However, although this route may be specialised for movements in the spatial domain it may be less efficient for movement timing control, thus explaining the stronger variability during the VS and VC tasks. The stronger right-sided dPMC activation for the VS condition may be a result of the strong bilateral activation in the visual cortex, where the pacing stimulus is processed. This bilateral visual activation may trigger the timing of the finger movements by transferring the sensory pacing codes via the left and right 'dorsal' pathways (via the dorsal frontoparietal path) to the prefrontal cortex, where stimulus-response associations are realised. Because both hemispheres are involved in this sensorimotor integration process it may be that this evokes some kind of interference, as the left and right premotor areas receive and process information that concerns move- ment timing of the right index finger. This might be a further reason that visual pacing is generally more variable than auditory pacing. The stronger activation on the right caudal dPMC just in front of M1 together with stronger activation in the LPs during the VC condition might be related to the necessity of achieving a new association between an imagined trigger stimulus and the motor commands. This interpretation is related to findings linking the caudal part of the dPMC to the learning of visual conditional tasks both in monkeys and humans [17,33].

A further differential activation was found for the SMA which was more strongly activated during both auditory conditions. At this point numerous functions have been suggested for the SMA, including movement complexity, movement sequencing, self versus external triggered movements, as well as movement imagination to name only a few [46]. The SMA activation found in our study may further emphasise the different strategies with which auditory and visual tapping tasks are accomplished. It may be that during both auditory conditions finger movements are more strongly guided by an internal reference rhythm than the visual conditions. We hypothesise that even during the AS condition the subjects used an internal rhythm to guide their movements. Thus, there is no principle difference between the paced (AS) and non-paced (AC) condition because in both conditions similar strategies are employed leading to similar brain activations. The external pacing stimulus is used rather as a time frame which is adopted by the motor control system at the beginning of the experimental sessions in order to establish a selfgenerated rhythm at the prescribed frequency. This internal self-generated movement rhythm may then be controlled by the SMA as suggested by findings emphasising the SMA as a key structure for controlling self-triggered movements. During the VS condition the visual pacing stimuli are so dominant that they may prevent the motor control system adopting its own movement rhythm. Therefore, the motor system is forced to rely on these external pacing stimuli. Because the VS condition does not support the generation of an internal rhythm, the following VC conditions are not able to rely on an established internal rhythm. Therefore the neural system might try to cope with this situation however inefficiently.

\subsection{Cerebellum}

The auditory conditions evoked stronger activations in the right inferior cerebellum (paramedian lobule and crus II). It is known from anatomical studies that there are two independent somatosensory maps in the cerebellum. A primary sensory homunculus is present in the superior cerebellar lobe ( $\mathrm{H} \mathrm{V}$ and $\mathrm{H} \mathrm{VI}$ ) and there is an independent bilateral representation in the inferior lobe (H VIIA and $\mathrm{H}$ VIIB) [47]. The activations found in our auditory conditions are located precisely at the second somatosensory 
hand representation in the inferior lobule ( $\mathrm{H}$ VIIA and $\mathrm{H}$ VIIB) while the activations in the superior-anterior cerebellum found during the visual condition are located in the primary somatosensory hand representation $(\mathrm{H} \mathrm{V}$ and $\mathrm{H}$ VI). Thus, our activation pattern suggests that the auditory and visual conditions evoke neural activity in more or less independent cerebellar neural networks associated with the control of finger movement. To interpret these results, we draw on the neuroanatomical literature derived from animal studies. Evidence from horseradish perioxidasetracing experiments in monkeys suggests that mossy fibre afferents to the posterior paramedian cerebellar lobe (i.e., $\mathrm{H}$ VIIA and H VIIB) are derived from the lateral portions of the pontine nuclei, whereas afferents to the anterior and posterior quadrangular lobules, as well as the superior semilunar lobule $(\mathrm{H} \mathrm{V}, \mathrm{H}$ VI, and superior $\mathrm{H}$ VIA, respectively) are derived from more medial pontine regions. The lateral pontine nuclei, in turn, receive much of their input from temporal and parietal association areas of the cerebral cortex, and the medial pontine nuclei receive their input from frontal but also from occipital areas [47]. Based on this evidence, one may assume that the inferiorposterior cerebellar activation in lobule HVIIA and HVIIB, which is only evident during the auditory conditions, may rely on auditory input and the concomitant activation in temporal lobe auditory cortices. On the other hand, superior-anterior cerebellar activations during the visual conditions may rely on the medial pontine pathway, which receives input from the visual system (during the visual pacing condition) situated in the occipital cortex. Thus, we suggest that the same processes are active in both auditory conditions (AS and $\mathrm{AC}$ ), causing activation in the right inferior-posterior cerebellar lobule. We speculate that during the AS and AC conditions, the motor system is switching over to a kind of 'auditory' motor control mode during which the inferior cerebellum is most active. The inferior cerebellar region has also been shown to be activated when explicit motor behaviour is required in contrast to the more superior activation during time perception tasks as well as during timed motor responses [21]. This argument would suggest that the visual and auditory conditions are principally controlled via different modes: one mode which is placing more emphasis on the perceptual aspect of the movement task while the other mode (the auditory one) is placing more emphasis on the motor aspect of the movement task.

While the auditory conditions generally evoke stronger activation in the right inferior cerebellum the visual conditions generally evoke stronger activation in the superior vermis region. Activation in this region has been related to the production of timed motor responses, particularly when it is complex and/or novel [36]. Following on from this, one may suggest that the visual conditions are more complex than the auditory conditions, thus requiring additional vermis activation.

\subsection{M1 and S1 activation}

The VC condition evoked stronger hemodynamic responses in the contralateral M1/S1 area, extending slightly into the LPi region. These 'extra' activations may be accounted for by the increased executive control required by the more difficult non-paced visual task. The difficulty in the latter might stem from the fact that the absence of the visual pacing stimulus does not allow the precise erection of stimulus-response associations. Because internal movement pacing possibly requires constant relearning or adaptation to the estimated or imagined pacing stimuli, mainly because imaging of stimuli is not easy, it is possible that somatosensory feedback of the fingers is constantly used to adjust tapping frequency. Thus, during the VC condition, finger movements are controlled in a more closed-loop manner in which motor commands are continuously compared with afferent information from the somatosensory senses. During the VS condition this afferent information may be not so important because the external pacing stimuli are the main constraints according to which the finger movement frequency has to be adjusted. Thus, it seems likely that the VS condition is associated with more computational demands in the dPMC areas while the $\mathrm{VC}$ condition is associated with more computational demands in M1 and S1.

\subsection{Deactivations in the sensory areas}

Interestingly, we found in the left and right anterior auditory areas comprising Heschl's gyrus and the anterior part of the planum temporale, remarkable deactivations during the AC conditions relative to baseline. Exactly the same areas are, however, significantly activated during the AS condition. Similarly we found decreased hemodynamic responses in the visual cortices during the VC conditions for areas that revealed strong signal increases in the VS condition. We hypothesise that the decreased activation during the non-paced conditions reflects decreased neuronal activity which might indicate decreased sensitivity to external stimuli. This interpretation corresponds with a recent computational work demonstrating that decreased neural activation increases effective post-synaptic membrane time constants, and selectively diminishes stimulusinduced dynamics [4]. It may be that when the external pacing stimulus is absent and the organism has to generate its own pacing stimulus via the fronto-parietal-cerebellar network, additional stimulation needs to be eliminated or attenuated to prevent disturbing input.

\section{Conclusion}

The main finding of the present study is a principle difference in terms of performance measures and brain 
activations between the auditory and visual tapping tasks. The auditory tapping tasks (AS and AC) were generated with less intertap variability than the visual tasks, thus suggesting different control modes. In terms of brain activation the auditory tasks are accompanied with stronger activations in a network comprising bilaterally of the vPMC, the left SMA, the right inferior cerebellum, and the left thalamus. This network also suggests that the paced finger-tapping task in the context of auditory pacing stimuli is controlled in a different way. This control mode can be best described as being driven by an internal movement rhythm closely matching the external pacing rhythm. The visual conditions on the other hand are typically accompanied by activations in the right LPi area and vermis region. In addition, there were substantial differences between visual synchronisation and continuation conditions in terms of stronger activation of the right dPMC and the right vPMC (for VS) and the left caudal dPMC, the left M1, the left S1, and the left and right LPi (for VC). We suggest that this activation pattern is due to the fact that the visual pacing stimulus is too demanding and dominant in order to support the generation of an internal movement rhythm, thus forcing the subjects to rely on external pacing. Because the visual synchronisation condition does not support the establishment of an internal movement rhythm the visual continuation rhythm relies on an inefficient and computationally demanding control mode, which requires, in particular, caudal dPMC, M1, S1, as well as bilateral LPi activation. Finally, there were no activations in the auditory and visual cortices during the continuation conditions when the pacing stimulus was absent, suggesting that internal pacing is not driven by activations in these areas evoked by 'imagined' pacing stimuli.

\section{Acknowledgements}

This study was financed by the Deutsche Forschungsgemeinschaft (DFG JA 737/7-1 and JA 737/7-2).

\section{References}

[1] T.J. Anderson, I.H. Jenkins, D.J. Brooks, M.B. Hawken, R.S. Frackowiak, C. Kennard, Cortical control of saccades and fixation in man. A PET study, Brain 117 (1994) 1073-1084.

[2] G. Aschersleben, W. Prinz, Synchronizing actions with events: the role of sensory information, Percept. Psychophys. 57 (1995) 305317.

[3] C.J. Bruce, M.E. Goldberg, M.C. Bushnell, G.B. Stanton, Primate frontal eye fields. II. Physiological and anatomical correlates of electrically evoked eye movements, J. Neurophysiol. 54 (1985) 714-734.

[4] D. Chawla, E. Lumer, K.J. Friston, The relationship between synchronization among neuronal populations and their mean activity levels, Neural Comput. 11 (1999) 1389-1411.
[5] A.C. Evans, D.L. Collins, S.R. Mills, E.D. Brown, R.L. Kelly, T.M. Peters, 3D statistical neuroanatomical models from 305 MRI volumes, in: Proc. IEEE Nuclear Science Symposium and Medical Imaging, 1993, pp. 1813-1817.

[6] G.R. Fink, R.S. Frackowiak, U. Pietrzyk, R.E. Passingham, Multiple nonprimary motor areas in the human cortex, J. Neurophysiol. 77 (1997) 2164-2174.

[7] L. Fogassi, V. Gallese, L. Fadiga, G. Luppino, M. Matelli, G. Rizzolatti, Coding of peripersonal space in inferior premotor cortex (area F4), J. Neurophysiol. 76 (1996) 141-157.

[8] P.T. Fox, J.M. Fox, M.E. Raichle, R.M. Burde, The role of cerebral cortex in the generation of voluntary saccades: a positron emission tomographic study, J. Neurophysiol. 54 (1985) 348-369.

[9] K.J. Friston, P. Jezzard, R. Turner, Analysis of functional MRI time-series, Hum. Brain Mapp. 1 (1994) 153-171.

[10] K.J. Friston, C.D. Frith, R. Turner, R.S. Frackowiak, Characterizing evoked hemodynamics with fMRI, Neuroimage 2 (1995) 157-165, [In Process Citation].

[11] K.J. Friston, A.P. Holmes, J.B. Poline, P.J. Grasby, S.C.R. Williman, R.S.J. Frackowiak, R. Turner, Analysis of fMRI time-series revisited, Neuroimage 2 (1995) 45-53.

[12] K.J. Friston, A.P. Holmes, K.P. Worsley, J.-B. Poline, C.D. Frith, R.S.J. Frackowiak, Statistical parametric maps in functional imaging: a general linear approach, Hum. Brain Mapp. 2 (1995) 189210.

[13] S.T. Grafton, R.P. Woods, M. Tyszka, Functional imaging of procedural motor learning: relating cerebral blood flow with individual subject performance, Hum. Brain Mapp. 1 (1994) 221-234.

[14] M.S. Graziano, X.T. Hu, C.G. Gross, Visuospatial properties of ventral premotor cortex, J. Neurophysiol. 77 (1997) 2268-2292.

[15] U. Halsband, N. Ito, J. Tanji, H.J. Freund, The role of premotor cortex and the supplementary motor area in the temporal control of movement in man, Brain 116 (1993) 243-266.

[16] M. Iacoboni, R.P. Woods, J.C. Mazziotta, Bimodal (auditory and visual) left frontoparietal circuitry for sensorimotor integration and sensorimotor learning, Brain 121 (1998) 2135-2143.

[17] M. Iacoboni, R.P. Woods, G. Lenzi, J.C. Mazziotta, Merging of oculomotor and somatomotor space coding in the human right precentral gyrus, Brain 120 (1997) 1635-1645.

[18] R. Ivry, S.W. Keele, Timing functions of the cerebellum, J. Cogn. Neurosci. 1 (1989) 136-152.

[19] R.B. Ivry, S.W. Keele, H.C. Diener, Dissociation of the lateral and medial cerebellum in movement timing and movement execution, Exp. Brain Res. 73 (1988) 167-180.

[20] L. Jancke, N.J. Shah, S. Posse, M. Grosse-Ryuken, H.W. MullerGartner, Intensity coding of auditory stimuli: an fMRI study, Neuropsychologia 36 (1998) 875-883.

[21] M. Jueptner, L. Flerich, C. Weiller, S.P. Mueller, H.C. Diener, The human cerebellum and temporal information processing: results from a PET experiment, NeuroReport 7 (1996) 2761-2765.

[22] R. Kawashima, P.E. Roland, B.T. O'Sullivan, Functional anatomy of reaching and visuomotor learning: a positron emission tomography study, Cereb. Cortex 5 (1995) 111-122.

[23] P.A. Kolers, J.M. Brewster, Rhythms and responses, J. Exp. Psychol. Hum. Percept. Perform. 11 (1985) 150-167.

[24] M. Krams, M.F. Rushworth, M.P. Deiber, R.S. Frackowiak, R.E. Passingham, The preparation, execution and suppression of copied movements in the human brain, Exp. Brain Res. 120 (1998) 386398.

[25] K. Kurata, Premotor cortex of monkeys: set- and movement-related activity reflecting amplitude and direction of wrist movements, J. Neurophysiol. 69 (1993) 187-200.

[26] K. Kurata, Information processing for motor control in primate premotor cortex, Behav. Brain Res. 61 (1994) 135-142.

[27] K. Kurata, Site of origin of projections from the thalamus to dorsal 
versus ventral aspects of the premotor cortex of monkeys, Neurosci. Res. 21 (1994) 71-76.

[28] K. Kurata, D.S. Hoffman, Differential effects of muscimol microinjection into dorsal and ventral aspects of the premotor cortex of monkeys, J. Neurophysiol. 71 (1994) 1151-1164.

[29] K. Kurata, E. Hoshi, Reacquisition deficits in prism adaptation after muscimol microinjection into the ventral premotor cortex of monkeys, J. Neurophysiol. 81 (1999) 1927-1938.

[30] O. Larsell, J. Jansen, The comparative anatomy and histology of the cerebellum: the human cerebellum, cerebellar connections, and cerebellar cortex, in: University of Minnesota, Minneapolis, 1972.

[31] M. Matelli, G. Luppino, G. Rizzolatti, Architecture of superior and mesial area 6 and the adjacent cingulate cortex in the macaque monkey, J. Comp. Neurol. 311 (1991) 445-462.

[32] J. Mates, T. Radil, E. Poppel, Cooperative tapping: time control under different feedback conditions, Percept. Psychophys. 52 (1992) 691-704.

[33] A.R. Mitz, M. Godschalk, S.P. Wise, Learning-dependent neuronal activity in the premotor cortex: activity during the acquisition of conditional motor associations, J. Neurosci. 11 (1991) 1855-1872.

[34] D.J. O'Boyle, J.S. Freeman, F.W. Cody, The accuracy and precision of timing of self-paced, repetitive movements in subjects with Parkinson's disease, Brain 119 (1996) 51-70.

[35] R.E. Passingham, Premotor cortex: sensory cues and movement, Behav. Brain Res. 18 (1985) 175-185.

[36] V.B. Penhune, R.J. Zattore, A.C. Evans, Cerebellar contributions to motor timing: a PET study of auditory and visual rhythm reproduction, J. Cogn. Neurosci. 10 (1998) 752-765.

[37] M. Peters, Description and validation of a flexible and broadly usable hand preference questionnaire, Laterality 3 (1998) 77-96.

[38] T.H. Rammsayer, On dopaminergic modulation of temporal information processing, Biol. Psychol. 36 (1993) 209-222.

[39] T. Rammsayer, W. Classen, Impaired temporal discrimination in Parkinson's disease: temporal processing of brief durations as an indicator of degeneration of dopaminergic neurons in the basal ganglia, Int. J. Neurosci. 91 (1997) 45-55.
[40] S.M. Rao, D.L. Harrington, K.Y. Haaland, J.A. Bobholz, R.W. Cox, J.R. Binder, Distributed neural systems underlying the timing of movements, J. Neurosci. 17 (1997) 5528-5535.

[41] G. Rizzolatti, M.A. Arbib, From grasping to speech: imitation might provide a missing link: reply, Trends. Neurosci. 22 (1999) 152.

[42] G. Rizzolatti, M. Gentilucci, L. Fogassi, G. Luppino, M. Matelli, S. Ponzoni-Maggi, Neurons related to goal-directed motor acts in inferior area 6 of the macaque monkey, Exp. Brain Res. 67 (1987) 220-224.

[43] G. Rizzolatti, M. Gentilucci, R.M. Camarda, V. Gallese, G. Luppino, M. Matelli, L. Fogassi, Neurons related to reaching-grasping arm movements in the rostral part of area 6 (area 6a beta), Exp. Brain Res. 82 (1990) 337-350.

[44] G. Rizzolatti, L. Fadiga, M. Matelli, V. Bettinardi, E. Paulesu, D. Perani, F. Fazio, Localization of grasp representations in humans by PET: 1. Observation versus execution, Exp. Brain Res. 111 (1996) 246-252.

[45] G. Rizzolatti, L. Fadiga, V. Gallese, L. Fogassi, Premotor cortex and the recognition of motor actions, Brain Res.Cogn. Brain Res. 3 (1996) 131-141.

[46] P.E. Roland, K. Zilles, The developing European computerized human brain database for all imaging modalities, Neuroimage 4 (1996) S39-47, [In Process Citation].

[47] J.D. Schmahmann, From movement to thought: anatomical substrates of the cerebellar contribution to cognitive processing, Hum. Brain Mapp. 4 (1995) 174-198.

[48] V. Sergent, J.B. Hellige, B. Cherry, Effects of responding hand and concurrent verbal processing on time-keeping and motor-implementation processes, Brain Cogn. 23 (1993) 243-262.

[49] K.M. Stephan, G.R. Fink, R.E. Passingham, D. Silbersweig, A.O. Ceballos-Baumann, C.D. Frith, R.S. Frackowiak, Functional anatomy of the mental representation of upper extremity movements in healthy subjects, J. Neurophysiol. 73 (1995) 373-386.

[50] A. Wing, A. Kristofferson, Response delays and the timing of discrete motor responses, Percept. Psychophys. 14 (1973) 5-12. 